\title{
Los pacientes y la sociedad han cambiado. Y nosotros ¿nos estamos adaptando al cambio?
}

\author{
Patients and Society Have Changed. Are We As Physicians Adapting to Change?
}

\author{
Pilar Román Sánchez ${ }^{1,2}$ \\ ${ }^{1}$ Servicio de Medicina Interna del Hospital de Requena. Valencia. ${ }^{2}$ Presidenta de la Sociedad Española de Medicina Interna.
}

Nuestro sistema nacional de salud, de cobertura universal y accesibilidad equitativa, es uno de los logros del estado del bienestar más significativo y más apreciado por los ciudadanos.

Los hospitales actuales nacieron hace 100 años y se organizaron en base a la especialización y el conocimiento, orientados a pacientes agudos, con la cama como recurso clave. Esta organización ha sido capaz de generar un gran avance científicotécnico que ha contribuido, junto al mayor nivel de vida, a la reducción de la mortalidad y a un aumento considerable de la expectativa de vida. Sin embargo, este avance continuo ha propiciado la subespecialización progresiva con la consiguiente fragmentación de los servicios.

En las últimas décadas, además, se han producido una serie de cambios que van a condicionar una nueva organización de la asistencia hospitalaria:

- El éxito en el tratamiento de muchas enfermedades agudas ha propiciado el auge de las patologías crónicas. Junto a la mayor esperanza de vida ha condicionado un cambio demográfico sustancial. El 60\% de los mayores de 65 años (colectivo que representa el 17,1\% de la población actual y, según estimaciones, el 19,2\% en 2.020 y más del 30\% en 2.050) padecen alguna enfermedad crónica y un porcentaje considerable de ellos padecen varias al mismo tiempo, ocasionando la mayor parte de los costes sanitarios. La enfermedad crónica es, actualmente, el patrón de morbilidad más importante de los países civilizados y la principal causa de mortalidad y discapacidad. Más del $70 \%$ de la actividad sanitaria está dedicada a la atención de los pacientes crónicos. Si no se reorienta la asistencia para este tipo de pacientes, está en juego la sostenibilidad del sistema sanitario.

- El coste sanitario ha crecido por encima del Producto Interior Bruto en los últimos años. Las causas son varias: la introducción de nuevas tecnologías sanitarias y el coste continuo de su renovación, los costes crecientes de la administración, la escasa integración entre profesionales y niveles de asistencia, la variabilidad clínica, la medicina defensiva y la mayor utilización de los servicios de salud debido a las enfermedades crónicas, la menor tolerancia a la enfermedad y la medicalización de problemas no sanitarios.

- Crisis económica grave con la consecuente necesidad de restricciones financieras importantes debido al descenso de los ingresos públicos que pone en situación de insolvencia al sistema sanitario.

- Pacientes cada vez más exigentes e informados que consideran la salud como un bien de consumo más, que piden información y tomar decisiones sobre su tratamiento. Internet ha puesto a su disposición información abundante sobre la salud. El 35\% de los españoles buscan información relativa a la salud de forma habitual, siendo los mayores de 65 años los mayores usuarios. Según una encuesta reciente, el $72 \%$ de las personas desearían que el médico les recomendasen lugares fiables para consultar información sanitaria y poder disponer de un blog o página donde comunicarse con los profesionales sanitarios.

- El desarrollo de las nuevas tecnologías con posibilidad de comunicación inmediata mediante teléfonos móviles inteligentes, correo electrónico, redes sociales, dispositivos de control de patologías en domicilio, telediagnóstico etc.

Frente a estos retos, más o menos acusados en los diferentes países occidentales, se van desarrollando nuevas tendencias sobre la función que deben adoptar los hospitales:

- Existe una creciente evidencia a favor de un cambio radical en el modelo asistencial para hacer frente a la epidemia de la cronicidad. Los profesionales del hospital forman equipos asistenciales con los de atención primaria y sociosanitaria para, de una manera proactiva, adelantarse a las descompensaciones de los pacientes con el objetivo de evitar progresión de la enfermedad, hospitalizaciones y dependencia, con segmentación de los pacientes según riesgos y atención multidisciplinar individualizada. Al mismo tiempo se hace necesario que los pacientes o sus cuidadores adopten una actitud activa y responsable sobre su enfermedad participando 


\section{En las últimas décadas, se han producido una serie de cambios que van a condicionar una nueva organización de la asistencia hospitalaria}

de las decisiones y comprometiéndose en los objetivos. Todo ello precisa de una redistribución de los roles y las competencias profesionales. La continuidad de la asistencia y la atención integral son objetivos fundamentales.

- El hospital abandona su papel centrípeto y se abre a la comunidad formando parte de una organización integrada, más flexible y abierta, extendiendo su actividad más allá de sus propios límites físicos. La tendencia a la ambulatorización de los procesos supondrá que los pacientes ingresados serán los de alta complejidad, los críticos, los de diagnóstico desconocido y complejo o los que precisen de alta tecnología o cirugía. Dispondrá de múltiples recursos asistenciales (camas, plazas de hospital de día, salas de intervenciones, hospitalización a domicilio, consultas de diagnóstico rápido, telemedicina, telemonitorización, historia personal de salud accesible en cualquier punto etc.), con una atención multidisciplinar y, en muchos casos, virtual debido al impacto de las tecnologías de la información.

- Los indicadores se basarán en resultados en salud, calidad y seguridad del paciente. La transparencia y la competencia entre los centros serán los medios de conseguir la mayor eficiencia.

- La gestión clínica y el compromiso y participación de los profesionales serán imprescindibles para conseguir los mejores resultados. Las unidades de gestión clínica en base a problemas de salud, en lugar de especialidades, se ha mostrado como la mejor fórmula para la asistencia de calidad centrada en el paciente.

$Y$, en este escenario, los internistas nos encontramos en una situación privilegiada. Nuestra formación generalista con visión integral del paciente nos hace los especialistas más adecuados para el cuidado de los pacientes crónicos complejos, formando equipo con los profesionales de atención primaria y evitando la fragmentación de la asistencia, las duplicidades y la medicación inadecuada.

Además, debemos ser los tutores de los pacientes durante su paso por el hospital, allá dondequiera que se encuentren. La mayor parte de los pacien- tes que se someten a intervenciones quirúrgicas padecen una o varias enfermedades crónicas. La atención a los problemas médicos de los pacientes quirúrgicos por parte de internistas ha demostrado ser eficiente, evitando descompensaciones y complicaciones.

Y somos, también, los expertos apropiados para el diagnóstico del paciente con enfermedad incierta no órgano-específica.

El reto es importante e ilusionante. Hemos de liderar el cambio de modelo asistencial formándonos en los que han demostrado éxito para el tratamiento de la cronicidad; en formar y liderar equipos multidisciplinares excelentes; en gestión clínica; en medicina perioperatoria; en el uso de las nuevas tecnologías; en calidad y seguridad del paciente y en la gestión del tiempo. No será fácil pero hemos de abandonar el valle de la comodidad si queremos ocupar el lugar que la medicina interna se merece y, sobretodo, si queremos dar a los pacientes la asistencia que se merecen, preservando, al mismo tiempo, nuestro sistema sanitario.

\section{Bibliografía}

1. Ham C., Dixon A., Brooke. B. Transforming the delivery of health and social care. The case for fundamental change. The King's Fund 2012

2. La sanidad pública ante la crisis. Recomendaciones para una actuación pública sensata y responsable. Documento de Debate. Asociación de Economía de la Salud.

3. Hernández Rodríguez M.A., Abaurrea Ortiz P., de Dios González Caballero J y cols. Sostenibilidad del Sistema Nacional de Salud: propuestas desde la Medicina de Familia

4. Redesigning health in Europe for 2020. eHealth Task Force Report. European Union, Healthcare 2012

5. Estrategia para el Abordaje de la Cronicidad en el Sistema Nacional de Salud Sanidad 2012. Ministerio de sanidad, servicios sociales e igualdad.

6. Hospitals and Care Systems of the Future. Committee on Performance Improvement of the American Hospital Association. September 2009

7. Healthcare 2015: Win-win or lose-lose? A portrait and a path to successful transformation. IBM Institute for Business Value. 2010

8. Bengoa R, Nuño Solinis R. Curar y cuidar. Innovación en la gestión de enfermedades crónicas: una guía para avanzar. Elsevier- Mason, 2008.

9. El hospital del futuro: las ideas de los expertos. Club GerTech. Madrid Network. Cluster Salud y Bienestar. 2011

10. Gil V, Barrubés J, Álvarez JC, Portella, E. La sostenibilidad financiera del sistema sanitario: 10 medidas estructurales para afrontar las causas del crecimiento del gasto. Barcelona: Antares Consulting; 2010.

11. Barrubés J., Carrillo E., Portella E. Repensando el hospital. Motores de cambio y respuestas innovadoras.

12. Corrigan P, Mitchell C. The hospital is dead, long life to the hospital. Reform, September 2011.

13. Malone TW. The Age of Hyperespecialization. Harvard Business Review, 2011; JulyAugust: $57-65$ 